\title{
Binding of Bile Salts to an Amphiphilic Polysulfonate Modified with Cholesterol Moieties
}

\author{
Shin-ichi YusA, ${ }^{1, \dagger}$ Kasumi IKedA, ${ }^{1}$ Tohei Yamamoto, ${ }^{1}$ and Yotaro MorishimA ${ }^{2}$ \\ ${ }^{1}$ Department of Materials Science and Chemistry, Graduate School of Engineering, University of Hyogo, \\ 2167 Shosha, Himeji 671-2201, Japan \\ ${ }^{2}$ Faculty of Engineering, Fukui University of Technology, 6-3-1 Gakuen, Fukui 910-8505, Japan
}

(Received January 21, 2005; Accepted May 9, 2005; Published August 15, 2005)

\begin{abstract}
A hydrophobically modified polysulfonate bearing a small mole percent $(5 \mathrm{~mol} \%)$ of cholesteryl moieties forms an intermolecularly bridged flower micelle in water, arising from intermolecular associations of the polymer-bound cholesteryl groups. Binding of sodium cholate (SC) to this polymer micelle in water was investigated using ${ }^{1} \mathrm{H}$ NMR spin-spin relaxation time, static light scattering, quasi-elastic light scattering, and fluorescence techniques. The intermolecular association of the polymer-bound cholesteryl groups was disrupted, to some extent, by the addition of SC, resulting in the formation of complexes of the polymer-bound cholesteryl groups and SC. To investigate interactions between the polymer-bound cholesteryl groups and SC, the cholesteryl groups were labeled with a naphthalene moiety. Fluorescence spectra of the naphthalene labels and NMR spin-spin relaxation time data suggested that interactions between the aggregates of the polymer-bound cholesteryl groups and SC occurred below a critical micelle concentration of SC in water. [DOI 10.1295/polymj.37.571]

KEY WORDS Bile Salt / Amphiphilic Polyelectrolyte / Cholesterol / Micelle / Hydrophobic Interaction / Fluorescence /
\end{abstract}

Bile salts are amphiphilic molecules consisting of a steroid backbone stored in the bile of all mammals. Bile salts can solubilize hydrophobic compounds by the formation of micelles. ${ }^{1}$ Therefore, bile salts play an important role in the emulsification and transport of dietary fat and lipids in food. Bile salts are synthesized from cholesterol in the liver to make up for a small daily loss during the enterohepatic circulation of bile salts. The biosynthesis of bile salts is the main process of cholesterol consumption. ${ }^{2,3}$ It is known that transmembrane absorption of bile salts occurs mainly by active transport of monomeric bile salts. ${ }^{4}$ If monomeric bile salts are adsorbed by hydrophobically modified polyelectrolytes in ileum, the enterohepatic circulation of bile salts may be changed. Namely, the amphiphilic polyelectrolytes may reduce or prevent the reabsorption of bile salts in ileum, which may lead to a lower cholesterol level in blood.

Interactions of hydrophobically modified polyelectrolytes with surfactants below or above a critical micelle concentration $(\mathrm{cmc})$ have been extensively studied. In general, hydrophobic modifications strengthen the water-soluble polymer and surfactant interactions by providing hydrophobic sites to which surfactants bind preferentially..$^{5-7}$ These interactions are important in biological phenomena and commercial process. ${ }^{8,9}$

Previously, we reported that random copolymers of sodium 2-(acrylamido)-2-methylpropanesulfonate
(AMPS) and cholesterol bearing methacrylate with low contents of cholesterol bearing monomer units $(\leq 10 \mathrm{~mol} \%)$ showed a strong tendency for interpolymer association in water even at very low polymer concentrations $\left(C_{\mathrm{p}}\right) \cdot{ }^{10-12}$ The cholesterol-bearing amphiphilic random copolymers form a "closed" type ${ }^{13}$ multi-polymer flower micelle consisting of a number of hydrophobic microdomains (i.e., cores) in one polymer micelle. The mean aggregation number of the cholesteryl groups in one hydrophobic microdomain is very small. Such an intermolecularly bridged flower micelle formed from the cholesterol-bearing amphiphilic polyelectrolytes is stable not to be fragmented by external shear stress. However, the hydrophobic crosslinks formed from the cholesterol aggregates are easily disrupted by the addition of a nonionic surfactant such as hexaethylene glycol $n$-dodecyl monoether $\left(\mathrm{C}_{12} \mathrm{E}_{6}\right)$, forming a polymer-micelle complex. ${ }^{11}$ These previous findings suggest that the cholesterol-bearing amphiphilic polyelectrolyte may adsorb small molecules with amphiphilic nature.

Bile salts resemble cholesterol in chemical structure consisting of a steroid backbone. Thus, it may be expected that the cholesterol-bearing amphiphilic polyelectrolyte adsorbs bile salts effectively to the pendent cholesteryl moieties and prevents the excess reabsorption of bile salts in the intestines tract. Sodium cholate (SC) (Figure 1), one of the most common bile salts, consists of a steroid backbone, a carboxyl, and three

${ }^{\dagger}$ To whom correspondence should be addressed (E-mail: yusa@eng.u-hyogo.ac.jp). 
hydroxyl groups. The association behavior of SC has been studied by using different experimental techniques such as light scattering, ${ }^{14} \mathrm{X}$-ray, ${ }^{15}$ and NMR. ${ }^{16}$

In the present paper, we report on the characterization of the binding of SC in water to an intermolecularly bridged flower micelle formed from the cholesterol-bearing amphiphilic polyelectrolytes. To investigate the interaction between the pendent cholesteryl moieties of the amphiphilic polyelectrolytes and SC with fluorescence techniques, the pendent cholesteryl moieties are labeled with a naphthalene moiety. For fluorescence studies, naphthalene-labeled polymers (p(AMPS/CholNp) and $\mathrm{p}(\mathrm{AMPS} / \mathrm{Np})$ in Figure 1) were employed. Furthermore, the interaction of the intermolecularly bridged flower micelle with SC was investigated by ${ }^{1} \mathrm{H}$ NMR relaxation time, static light scattering (SLS), quasi-elastic light scattering (QELS), and fluorescence in water.
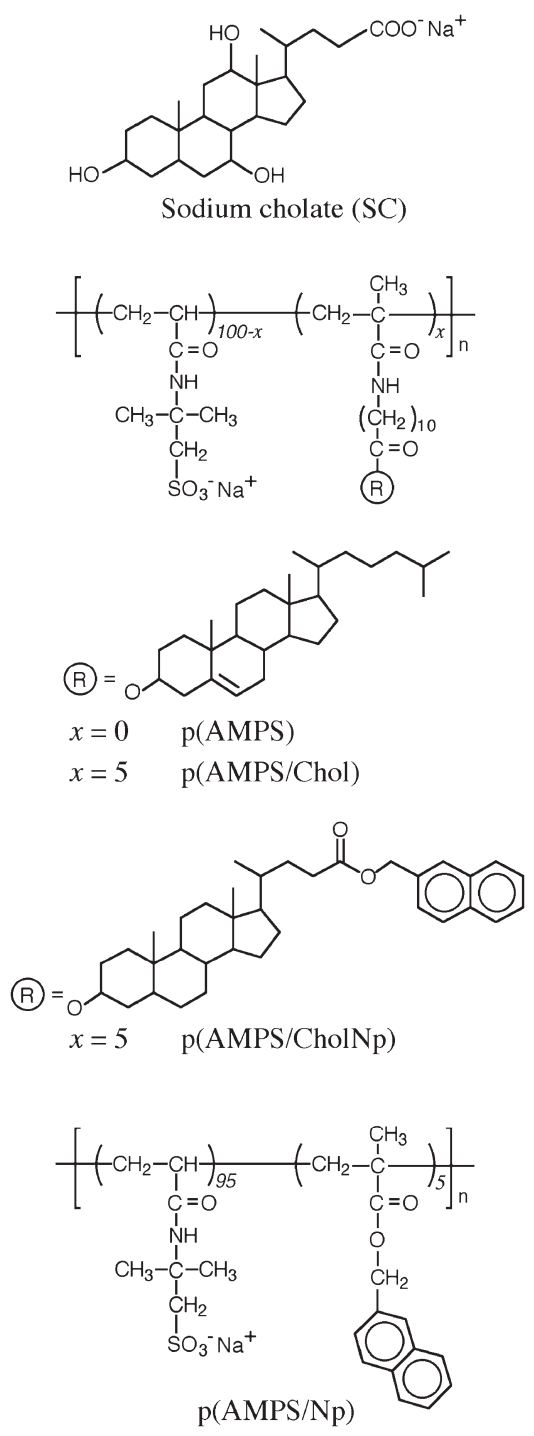

Figure 1. Chemical structures of sodium cholate (SC) and polymers used in this study.

\section{EXPERIMENTAL}

\section{Materials}

Potassium $3 \alpha$-hydroxy- $5 \beta$-cholanoate was prepared by neutralization of the respective acids $(25.0 \mathrm{~g}, 66.4$ mmol) with an equivalent of potassium hydroxide $(3.90 \mathrm{~g}, 70.0 \mathrm{mmol})$ in methanol followed by precipitation of the salts with diethyl ether: yield $26.3 \mathrm{~g}$ (95.5\%). 11-Methacrylamidoundecanoic acid was prepared according to the method reported by Gan and co-workers. ${ }^{17}$ 2-(Acrylamido)-2-methylpropanesulfonic acid (AMPS) and SC, purchased from Tokyo Kasei Kogyo Co., were used without further purification. 2,2'-Azobis(isobutyronitrile) (AIBN) was recrystallized from methanol. $N, N$-Dimethylformamide (DMF) was dried over calcium hydride and distilled under reduced pressure. The cholesteryl moiety ( $5 \mathrm{~mol} \%$ ) bearing random copolymer ( $\mathrm{p}$ (AMPS/Chol) in Figure 1) and sodium 2-(acrylamido)-2-methylpropanesulfoante homopolymer (p(AMPS) in Figure 1) employed in this study are the same polymers as those used in our previous work. ${ }^{12}$ Water was purified with a Millipore Milli-Q system. Other reagents were used as received.

\section{2-Naphthylmethylmethacrylate}

A solution of potassium methacrylate $(9.40 \mathrm{~g}, 75.8$ mmol), 2-(bromomethyl)naphthalene $(5.60 \mathrm{~g}, 25.3$ $\mathrm{mmol})$, and tetra- $n$-butylammonium bromide $(1.60 \mathrm{~g}$, $5.10 \mathrm{mmol})$ in water $(152 \mathrm{~mL})$ and chloroform $(75.8$ $\mathrm{mL}$ ) was heated to $110-115^{\circ} \mathrm{C}$ in an oil bath for $45 \mathrm{~h}$. The mixture was diluted with chloroform $(500 \mathrm{~mL})$, washed twice with water, and dried over anhydrous $\mathrm{Na}_{2} \mathrm{SO}_{4}$. The solvent was removed by evaporation, and the crude product was purified by chromatography on a silica gel column with chloroform as an eluent. The second fraction was collected, and the concentration gave a white solid: yield $774 \mathrm{mg}(13.5 \%)$; m.p. $63-65{ }^{\circ} \mathrm{C} ;{ }^{1} \mathrm{H}$ NMR $\left(270 \mathrm{MHz}, \mathrm{CDCl}_{3}\right): \delta 1.98(\mathrm{~s}$, $3 \mathrm{H}), 5.34(\mathrm{~s}, 2 \mathrm{H}), 5.59$ (bs, $1 \mathrm{H}), 6.18$ (bs, 1H), 7.44-7.49 (m, 3H), 7.81-7.84 (m, 4H). ${ }^{13} \mathrm{C}$ NMR (DEPT, $\left.67.8 \mathrm{MHz}, \mathrm{CDCl}_{3}\right): \delta 18.39\left(\mathrm{CH}_{3}\right), 66.52$ $\left(\mathrm{CH}_{3}\right), 125.75(\mathrm{CH}), 125.84\left(\mathrm{CH}_{2}\right), 126.18(\mathrm{CH})$, $126.24(\mathrm{CH}), 127.13(\mathrm{CH}), 127.67(\mathrm{CH}), 127.94$ $(\mathrm{CH}), 128.32(\mathrm{CH}), 133.05$ (quaternary), 133.15 (quaternary), 133.50 (quaternary), 136.21 (quaternary), 167.22 (quaternary).

\section{$3 \alpha-H y d r o x y-5 \beta-\left(2^{\prime}\right.$-naphthylmethylcholanoate)}

A solution of potassium $3 \alpha$-hydroxy- $5 \beta$-cholanoate $(23.0 \mathrm{~g}, \quad 55.5 \mathrm{mmol}), \quad 2$-(bromomethyl)naphthalene $(8.18 \mathrm{~g}, 37.0 \mathrm{mmol})$, and tetra- $n$-butylammonium bromide $(2.38 \mathrm{~g}, 7.40 \mathrm{mmol})$ in water $(100 \mathrm{~mL})$ and chloroform $(40.0 \mathrm{~mL})$ was heated to $110-115^{\circ} \mathrm{C}$ in 
an oil bath for $40 \mathrm{~h}$. The mixture was diluted with chloroform $(500 \mathrm{~mL})$, washed twice with water, and dried over anhydrous $\mathrm{Na}_{2} \mathrm{SO}_{4}$. The solvent was removed by evaporation, and the crude product was purified by chromatography on a silica gel column with chloroform as an eluent. The second fraction was collected, and the concentration gave a white solid: yield $12.8 \mathrm{~g}$ (68.8\%); m.p. $178-181^{\circ} \mathrm{C}$; IR (KBr): 1187, 1719, 2864, 2929, $3491 \mathrm{~cm}^{-1} ;{ }^{1} \mathrm{H}$ NMR $\left(500 \mathrm{MHz}, \mathrm{CDCl}_{3}\right): \delta 0.60(\mathrm{~s}, 3 \mathrm{H}), 0.89-1.95(\mathrm{~m}$, $33 \mathrm{H}), 2.26-2.48(\mathrm{~m}, 2 \mathrm{H}), 3.54-3.65(\mathrm{~m}, 1 \mathrm{H}), 5.26$ (s, 2H), 7.42-7.50 (m, 3H), 7.80-7.84 (m, 4H). ${ }^{13} \mathrm{C}$ NMR (DEPT, $\left.125 \mathrm{MHz}, \mathrm{CDCl}_{3}\right): \delta 11.96\left(\mathrm{CH}_{3}\right)$, $18.23\left(\mathrm{CH}_{3}\right), 20.75\left(\mathrm{CH}_{2}\right), 23.34\left(\mathrm{CH}_{3}\right), 24.14$ $\left(\mathrm{CH}_{2}\right), 26.36\left(\mathrm{CH}_{2}\right), 27.14\left(\mathrm{CH}_{2}\right), 28.14\left(\mathrm{CH}_{2}\right)$, $30.48\left(\mathrm{CH}_{2}\right), 30.93\left(\mathrm{CH}_{2}\right), 31.26\left(\mathrm{CH}_{2}\right), 34.51$ (quaternary), $35.28(\mathrm{CH}), 35.30\left(\mathrm{CH}_{2}\right), 35.77(\mathrm{CH})$, $36.38\left(\mathrm{CH}_{2}\right), 40.09\left(\mathrm{CH}_{2}\right), 40.35(\mathrm{CH}), 42.03(\mathrm{CH})$, 42.67 (quaternary), $55.87(\mathrm{CH}), 56.41(\mathrm{CH}), 66.21$ $\left(\mathrm{CH}_{2}\right), 71.81(\mathrm{CH}), 125.90(\mathrm{CH}), 126.19(\mathrm{CH})$, $126.25(\mathrm{CH}), 127.32(\mathrm{CH}), 127.67(\mathrm{CH}), 127.93$ $(\mathrm{CH}), 128.32(\mathrm{CH}), 133.04$ (quaternary), 133.14 (quaternary), 133.48 (quaternary), 174.17 (quaternary).

$3 \alpha-\left[\left(11^{\prime}-\right.\right.$ Methacrylamido $)$ undecanoyloxy $]-5 \beta-\left(2^{\prime \prime}-\right.$ naphthylmethylcholanoate) (CholNp)

11-Methacrylamidoundecanoic acid $(4.12 \mathrm{~g}, 15.3$ mmol) and a trace of 2,6-di-tert-butyl-p-cresol were dissolved in thionyl chloride $(4.70 \mathrm{~mL})$, and the mixture was refluxed for $3 \mathrm{~h}$. An excess of thionyl chloride was then removed under reduce pressure. The crude product was dissolved in dry chloroform $(17.0 \mathrm{~mL})$, and the solution was added to a dry chloroform solution $(65.0 \mathrm{~mL})$ containing $3 \alpha$-hydroxy- $5 \beta$ ( $2^{\prime}$-naphthylmethylcholanoate $) \quad(6.55 \mathrm{~g}, \quad 12.4 \mathrm{mmol})$ and pyridine $(1.40 \mathrm{~g}, 17.7 \mathrm{mmol})$ over a period of $30 \mathrm{~min}$ at $0^{\circ} \mathrm{C}$. The solution was stirred for $19 \mathrm{~h}$ at room temperature, and then the solvent was removed, and the crude product was purified by chromatography on a silica gel column with chloroform as an eluent. The third fraction was collected, and the concentration gave a clear oil: yield $1.08 \mathrm{~g}(11.3 \%)$; IR (KBr): 1733, 2857, 2929, $3336 \mathrm{~cm}^{-1} ;{ }^{1} \mathrm{H}$ NMR (270 MHz, $\left.\mathrm{CDCl}_{3}\right)$ : $\delta 0.60(\mathrm{~s}, 3 \mathrm{H}), 0.89-1.96(\mathrm{~m}, 51 \mathrm{H}), 2.23-2.49(\mathrm{~m}$, $4 \mathrm{H}), 3.25-3.32(\mathrm{~m}, 2 \mathrm{H}), 4.68-4.76(\mathrm{~m}, 1 \mathrm{H}), 5.27(\mathrm{~s}$, 2H), 5.29 (s, 1H), 5.66 (s, 1H), 5.88 (bs, 1H), 7.44$7.50(\mathrm{~m}, 3 \mathrm{H}), 7.82-7.85(\mathrm{~m}, 4 \mathrm{H}) .{ }^{13} \mathrm{C}$ NMR (DEPT, $\left.67.8 \mathrm{MHz}, \mathrm{CDCl}_{3}\right): \delta 11.93\left(\mathrm{CH}_{3}\right), 11.97\left(\mathrm{CH}_{3}\right)$, $18.21\left(\mathrm{CH}_{3}\right), \quad 20.74 \quad\left(\mathrm{CH}_{3}\right), 23.20\left(\mathrm{CH}_{2}\right), 23.31$ $\left(\mathrm{CH}_{2}\right), 24.08\left(\mathrm{CH}_{2}\right), 25.00\left(\mathrm{CH}_{2}\right), 26.24\left(\mathrm{CH}_{2}\right)$, $26.62\left(\mathrm{CH}_{2}\right), 26.87(\mathrm{CH}), 26.94\left(\mathrm{CH}_{2}\right), 28.11\left(\mathrm{CH}_{2}\right)$, $29.02\left(\mathrm{CH}_{2}\right), 29.18\left(\mathrm{CH}_{2}\right), 29.27\left(\mathrm{CH}_{2}\right), 29.36$ $\left(\mathrm{CH}_{2}\right), 29.49\left(\mathrm{CH}_{2}\right), 30.91\left(\mathrm{CH}_{2}\right), 31.23\left(\mathrm{CH}_{2}\right)$, $32.22\left(\mathrm{CH}_{2}\right), \quad 34.50 \quad\left(\mathrm{CH}_{2}\right), \quad 34.68\left(\mathrm{CH}_{2}\right), 34.97$ $\left(\mathrm{CH}_{2}\right), 35.24(\mathrm{CH}), 35.69$ (quaternary), $39.62\left(\mathrm{CH}_{2}\right)$,
$40.04(\mathrm{CH}), 40.31$ (quaternary), $41.82\left(\mathrm{CH}_{2}\right), 42.63$ $(\mathrm{CH}), 55.90(\mathrm{CH}), 56.37(\mathrm{CH}), 66.17\left(\mathrm{CH}_{2}\right), 74.02$ $(\mathrm{CH}), 119.01\left(\mathrm{CH}_{2}\right), 119.19(\mathrm{CH}), 125.84(\mathrm{CH})$, $126.20(\mathrm{CH}), 127.26(\mathrm{CH}), 127.62(\mathrm{CH}), 127.89$ $(\mathrm{CH}), 128.27(\mathrm{CH}), 133.01$ (quaternary), 133.10 (quaternary), 133.44 (quaternary), 140.20 (quaternary), 168.34 (quaternary), 173.37 (quaternary), 174.07 (quaternary).

\section{Polymer}

A random copolymer of sodium salt of AMPS and CholNp (p(AMPS/CholNp)) was prepared by freeradical copolymerization initiated by AIBN in DMF. A procedure for the copolymerization is as follows. A predetermined amount of AMPS $(1.97 \mathrm{~g}, 9.50$ mmol) was neutralized by equimolar $\mathrm{Na}_{2} \mathrm{CO}_{3}$ in a 20.0-mL DMF solution. To this solution was added predetermined amounts of CholNp $(0.38 \mathrm{~g}, 0.50$ mmol) and AIBN $(4.20 \mathrm{mg}, 0.0260 \mathrm{mmol})$. The solution was placed into a glass ampul and outgassed on a vacuum line by six freeze-pump-thaw cycles, and then the ampul was sealed under vacuum. Polymerization was carried out at $60^{\circ} \mathrm{C}$ for $12 \mathrm{~h}$. The polymerization mixture was poured into a large excess of diethyl ether to precipitate the resulting polymer. The polymer was purified by reprecipitating from methanol into a large excess of diethyl ether three times. The polymer was then dissolved in water, and the aqueous solution was dialyzed against pure water for a week. The polymer was recovered by freeze-drying. A reference copolymer of AMPS and 2-naphtylmethylmethacrylate ( $\mathrm{p}(\mathrm{AMPS} / \mathrm{Np})$ ) was prepared and purified in a manner similar to the case of the copolymer described above. The contents of naphthalene moiety in $\mathrm{p}(\mathrm{AMPS} / \mathrm{CholNp}$ ) and $\mathrm{p}(\mathrm{AMPS} / \mathrm{Np})$ are $5 \mathrm{~mol} \%$ determined by ${ }^{1} \mathrm{H}$ NMR and UV-vis absorption spectroscopy.

\section{Measurements}

Infrared (IR) spectra were measured with $\mathrm{KBr}$ pellets on a JASCO FT/IR-5M spectrophotometer. UVvis absorption spectra were recorded on a Shimadzu UV-2500PC spectrophotometer.

Gel-permeation chromatography (GPC) measurements were performed with a Tosoh DP-8020 pump equipped with a Tosoh RI-8021 refractive index detector and two sets of Tosoh TSK gel $\alpha$-M columns using a $10-\mathrm{mM} \mathrm{LiBr}$ containing mixed solvent of dimethylsulfoxide (DMSO) and DMF (60/40, v/v) as an eluent at a flow rate of $1.0 \mathrm{~mL} / \mathrm{min}$. Sample solutions were filtered with a $0.45-\mu \mathrm{m}$ pore size membrane filter before measurements. The number-average molecular weight $\left(M_{\mathrm{n}}\right)$, weight-average molecular weight $\left(M_{\mathrm{w}}\right)$, and molecular weight distribution $\left(M_{\mathrm{w}} / M_{\mathrm{n}}\right)$ for the polymers were calibrated with standard poly- 
(ethylene oxide) samples.

Proton NMR spectra were obtained with a JEOL EX-270 spectrometer or Bruker DRX 500 NMR spectrometer using a deuterium lock at a constant temperature of $27^{\circ} \mathrm{C}$ during the whole run. Proton NMR spin-spin relaxation times $\left(T_{2}\right)$ were determined by a Carr-Purcell-Meiboon-Gill (CPMG) method. ${ }^{18}$ For $T_{2}$ measurements, NMR tubes containing $\mathrm{D}_{2} \mathrm{O}$ solutions were deaerated by purging with $\mathrm{Ar}$ gas for $30 \mathrm{~min}$.

Static light scattering (SLS) and quasi-elastic light scattering (QELS) data were obtained at $25^{\circ} \mathrm{C}$ with an Otsuka Electronics Photal DLS-7000DL light scattering spectrometer equipped with an ALV-5000E multi- $\tau$, digital time correlator at a fixed scattering angle $(\theta)$ of $90^{\circ}$. A $488 \mathrm{~nm} \mathrm{Ar}^{+}$laser was used as a light source and output power was $50.0 \mathrm{~mW}$. Sample solutions of polymers in $0.1 \mathrm{M} \mathrm{NaCl}$ were filtered with a $0.2-\mu$ pore size membrane filter before measurements. The intensity autocorrelation function $g^{(2)}(t)$ was obtained by QELS measurements. The intensity autocorrelation function is related to the normalized autocorrelation function $g^{(1)}(t)$, described as

$$
g^{(2)}(t)=B\left(1+\beta\left|g^{(1)}(t)\right|^{2}\right)
$$

where $\beta$ is a parameter of the optical system (constant) and $B$ is a baseline. The inverse Laplace transform (ILT) analysis of the normalized intensity autocorrelation functions, $g^{(2)}(t)$, was performed using the algorithm REPES to obtain the relaxation time distribution $(\tau A(\tau))$ according to ${ }^{19-21}$

$$
g^{(1)}(t)=\int \tau A(\tau) \exp (-t / \tau) \mathrm{d} \ln \tau
$$

where $g^{(1)}(t)$ is the normalized first-order electric field time correlation function, $\tau$ is the relaxation time. The relaxation time distribution is presented as a $\tau A(\tau)$ versus $\ln \tau$ profile, with $\tau A(\tau)$, providing an equal area representation. The diffusion coefficient $(D)$ is calculated from $D=\Gamma / q^{2}$, where $\Gamma=\tau^{-1}$. Here $q$ is the scattering vector, $q=(4 \pi n / \lambda) \sin (\theta / 2), n$ being the refractive index of the solvent and $\lambda$ the wavelength $(=488 \mathrm{~nm})$. The hydrodynamic radius $\left(R_{\mathrm{h}}\right)$ is calculated using the Einstein-Stokes relation $R_{\mathrm{h}}=k_{\mathrm{B}} T$ / $6 \pi \eta D$, where $k_{\mathrm{B}}$ is Boltzmann's constant, $T$ is the absolute temperature, and $\eta$ is the solvent viscosity. The details of QELS instrumentation and theory are described in the literature. ${ }^{22,23}$

Fluorescence spectra were recorded on a Hitachi F-4500 fluorescence spectrometer with excitation at $290 \mathrm{~nm}$ for the naphthalene label at room temperature. Excitation and emission slit widths were maintained at 25 and $1.5 \mathrm{~nm}$, respectively.
Table I. Molecular characteristics of polymers

\begin{tabular}{lccc}
\hline & $M_{\mathrm{n}} \times 10^{-4 \mathrm{a}}$ & $M_{\mathrm{w}} \times 10^{-5 \mathrm{a}}$ & $M_{\mathrm{w}} / M_{\mathrm{n}}{ }^{\mathrm{a}}$ \\
\hline $\mathrm{p}$ (AMPS/Chol) & 2.51 & 1.41 & 5.62 \\
$\mathrm{p}($ AMPS) & 2.05 & 1.08 & 5.29 \\
$\mathrm{p}($ AMPS/CholNp) & 2.00 & 1.03 & 5.17 \\
$\mathrm{p}($ AMPS/Np) & 1.69 & 0.98 & 5.78 \\
\hline
\end{tabular}

${ }^{a}$ Determined by GPC in a 10-mM LiBr DMSO/DMF (60/40, $\mathrm{v} / \mathrm{v}$ ) solution calibrated with poly(ethylene oxide) standards.

\section{RESULTS AND DISCUSSION}

All the polymers used in this study are completely soluble in water. Values of $M_{\mathrm{n}}, M_{\mathrm{w}}$, and $M_{\mathrm{w}} / M_{\mathrm{n}}$ for these polymers estimated by GPC are listed in Table I. As reported in our earlier paper, ${ }^{12}$ the cholesterol-bearing amphiphilic polyelectrolyte, p(AMPS/ Chol), forms a multipolymer aggregate consisting of a number of hydrophobic microdomains in one polymer aggregate. The mean aggregation number of the cholesteryl groups forming one hydrophobic microdomain was estimated to be $c a$. 12 by using a fluorescence quenching technique. This type of multipolymer aggregate can be envisaged as an intermolecularly bridged flower micelle. ${ }^{12}$

$\mathrm{SC}$ is known to form a micelle in water. ${ }^{24}$ To obtain information about the micellization of SC, we measured ${ }^{1} \mathrm{H}$ NMR spin-spin relaxation times $\left(T_{2}\right)$ at varying concentrations of $\mathrm{SC}$ ([SC]) in the presence and absence of $\mathrm{p}$ (AMPS/Chol). It is known that ${ }^{1} \mathrm{H}$ NMR relaxation times are influenced by changes in the dynamic motion of protons. Therefore, a critical micelle concentration $(\mathrm{cmc})$ for SC can be estimated by NMR relaxation times. We chose a resonance peak at $3.9 \mathrm{ppm}$ assigned to the methine proton in SC for $T_{2}$ measurements, because this peak is isolated from the other peaks. Figure 2 shows the variation of $T_{2}$ for $\mathrm{SC}$ as a function of [SC]. In the absence of

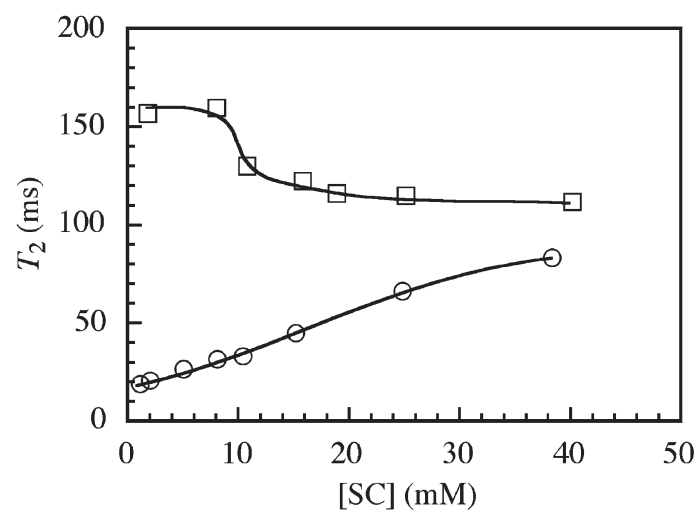

Figure 2. Plots of NMR spin-spin relaxation times $\left(T_{2}\right)$ estimated for a resonance peak around $3.9 \mathrm{ppm}$ due to $\mathrm{SC}$ in the absence $(\square)$ and presence of p(AMPS/Chol) $(\bigcirc)$ at $C_{\mathrm{p}}=5.0 \mathrm{~g} /$ $\mathrm{L}$ in $\mathrm{D}_{2} \mathrm{O}$ as a function of [SC]. 
$\mathrm{p}$ (AMPS/Chol), $T_{2}$ values exhibit a transition from 160 to $115 \mathrm{~ms}$ upon increase in [SC] from 8 to $16 \mathrm{mM}$. This transition of $T_{2}$ can be interpreted as being due to the micellization of SC. An SC concentration of $c a .10 \mathrm{mM}$ at which the transition of $T_{2}$ occurs is in good agreement with cmc values determined by different techniques such as potentiometry, ${ }^{25}$ surface tension, ${ }^{26}$ and dye solubilization. ${ }^{27}$ Therefore, it can be concluded that $T_{2}$ for SC in the monomer and micellar states are more or less 160 and $120 \mathrm{~ms}$, respectively.

In the presence of $\mathrm{p}$ (AMPS/Chol) $\left(C_{\mathrm{p}}=5.0 \mathrm{~g} / \mathrm{L}\right)$, the dependence of $T_{2}$ on [SC] is completely different from that in the absence of the polymer. Values of $T_{2}$ in the presence of the polymer are markedly smaller than $T_{2}$ values for SC micelles formed in the absence of the polymer, and there is no transition of $T_{2} . T_{2}$ exhibits a very small value of $20 \mathrm{~ms}$ at $[\mathrm{SC}]=1 \mathrm{mM}$ in the presence of the polymer, followed by a monotonic increase in $T_{2}$ with increasing [SC]. These observations indicate that motions of $\mathrm{SC}$ are strongly restricted, which suggests that $\mathrm{SC}$ is bound to a cholesterol aggregate in the micelle of $\mathrm{p}$ (AMPS/Chol). The binding of SC occurs regardless of whether its concentrations are below or beyond cmc. From the fact that $T_{2}$ increases with increasing $[\mathrm{SC}]$ in the whole range of [SC] shown in Figure 2, it is inferred that cholesterol aggregates in the polymer micelle are dissociated as a result of the binding of SC. It appears that $T_{2}$ for higher [SC] in the presence of the polymer gradually nears $T_{2}$ for SC micelles in the absence of the polymer.

The dissociation of the multipolymer micelle of p(AMPS/Chol) upon binding of SC was evidenced by light scattering data. Figure 3 shows variations of the excess Rayleigh ratios $\left(R_{90}\right)$ observed at a fixed scattering angle of $90^{\circ}$ for aqueous solutions of $5.0 \mathrm{~g} / \mathrm{L}$ p(AMPS/Chol) containing SC at different concentrations. The $R_{90}$ values are plotted against

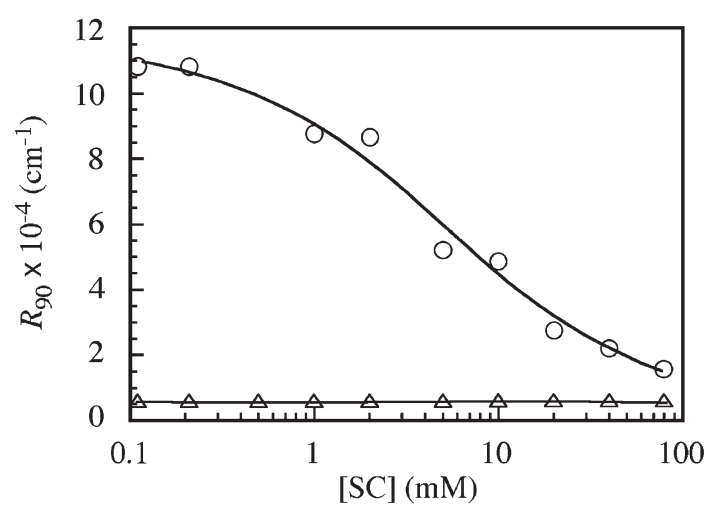

Figure 3. Excess Rayleigh ratios $\left(R_{90}\right)$ at a fixed scattering angle of $90^{\circ}$ for $0.1 \mathrm{M} \mathrm{NaCl}$ aqueous solutions of $\mathrm{p}$ (AMPS/Chol) $(\bigcirc)$ and p(AMPS) $(\triangle)$ at $C_{\mathrm{p}}=5.0 \mathrm{~g} / \mathrm{L}$ plotted as a function of [SC] at $25^{\circ} \mathrm{C}$.
[SC]. For comparison, $R_{90}$ values for $5.0 \mathrm{~g} / \mathrm{L}$ of $\mathrm{p}$ (AMPS) solutions containing SC at different concentrations are also plotted in the same figure. At low [SC] $(<0.2 \mathrm{mM})$, the $R_{90}$ values for $\mathrm{p}$ (AMPS/Chol) are large, because the copolymer forms intermolecularly bridged flower micelles. ${ }^{12}$ The $R_{90}$ values are proportionally related to the molecular mass for multipolymer aggregates. As $[\mathrm{SC}]$ is increased, $R_{90}$ for p(AMPS/Chol) decreases remarkably, indicating that the bridged flower micelle is disrupted by the addition of SC. In contrast, $R_{90}$ values for p(AMPS) are practically constant at much smaller values than those for $\mathrm{p}$ (AMPS/Chol) independent of [SC], which indicates that SC does not interact with $\mathrm{p}$ (AMPS).

Figure 4 compares QELS relaxation time distributions for aqueous solutions of $5.0 \mathrm{~g} / \mathrm{L}$ of p(AMPS/ Chol) containing $0.1 \mathrm{M} \mathrm{NaCl}$ in the absence and presence of $80 \mathrm{mM}$ of SC. The relaxation time distributions for the copolymer are bimodal with fast and slow relaxation modes. The $R_{\mathrm{h}}$ values calculated from the relaxation times at the peak top of the fast and slow mode distributions are 10.8 and $103 \mathrm{~nm}$, respectively. In the absence of SC, the peak for the fast mode relaxation time distribution can be ascribed to very small aggregates or unimers, and the slow mode is attributed to the intermolecularly bridged flower micelle formed from $\mathrm{p}$ (AMPS/Chol). ${ }^{10,11}$ When SC was added to the aqueous polymer solution, the relaxation times for both the fast and slow modes decreased. The apparent $R_{\mathrm{h}}$ values estimated from the fast and slow relaxation time distributions at $[\mathrm{SC}]=80 \mathrm{mM}$ are 6.11 and $74.0 \mathrm{~nm}$, respectively. The $R_{\mathrm{h}}$ value of the fast mode for $\mathrm{p}$ (AMPS/Chol) in the presence of SC is close to the $R_{\mathrm{h}}$ value for $\mathrm{p}$ (AMPS) in aqueous solution $(7.0 \mathrm{~nm}) .{ }^{11}$ The decrease in the $R_{\mathrm{h}}$ value of the slow

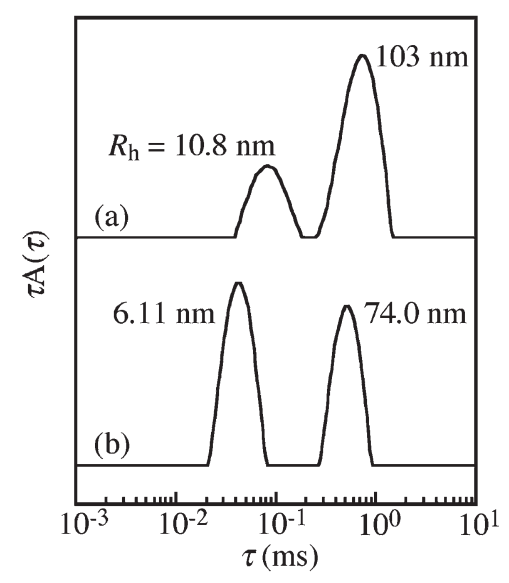

Figure 4. Distributions of the relaxation times in quasi-elastic light scattering (QELS) for $\mathrm{p}(\mathrm{AMPS} / \mathrm{Chol})$ at $C_{\mathrm{p}}=5.0 \mathrm{~g} / \mathrm{L}$ in $0.1 \mathrm{M} \mathrm{NaCl}$ aqueous solutions in the absence (a) and presence $(80 \mathrm{mM})$ (b) of $\mathrm{SC}$ at $25^{\circ} \mathrm{C}$, where the scattering angle is fixed at $90^{\circ}$. 
relaxation mode in the presence of SC indicates that the intermolecularly bridged flower micelle is dissociated upon binding of SC to the polymer-bound cholesteryl groups. It is worth noting that the relative peak area ratio of the fast mode to the slow mode in the presence of SC is larger than that in the absence of SC. This observation also indicates that some intermolecularly bridged flower micelles are disrupted by addition of SC.

To investigate interactions of the polymer-bound cholesterol with SC using a fluorescence technique, we prepared naphthalene-labeled copolymers (p(AMPS/CholNp) and $\mathrm{p}(\mathrm{AMPS} / \mathrm{Np}$ ) in Figure 1). In $\mathrm{p}$ (AMPS/CholNp), a naphthalene label is covalently linked to the side chain of the steroid-containing monomer unit in the vicinity of the steroid moiety. We have confirmed that the self-associative properties of $\mathrm{p}$ (AMPS/CholNp) in water are essentially the same as those of the non-labeled polymer, p(AMPS/Chol). Fluorescence spectra of naphthalene labels in p(AMPS/CholNp) exhibit an excimer emission peaking at $390 \mathrm{~nm}$. The $I_{\mathrm{e}} / I_{\mathrm{m}}$ ratios for $\mathrm{p}$ (AMPS/CholNp) were almost constant at 0.33 over a wide range of the polymer concentrations $\left(0.1<C_{\mathrm{p}}<10 \mathrm{~g} / \mathrm{L}\right)$, where $I_{\mathrm{e}}$ and $I_{\mathrm{m}}$ are the fluorescence intensities of excimer and monomer emissions at 335 and $390 \mathrm{~nm}$, respectively. In contrast, the $I_{\mathrm{e}} / I_{\mathrm{m}}$ ratios for the reference copolymer, $\mathrm{p}(\mathrm{AMPS} / \mathrm{Np})$, are much smaller and constant at 0.14 independent of $C_{\mathrm{p}}$ in water. In methanol, on the other hand, the $I_{\mathrm{e}} / I_{\mathrm{m}}$ ratios for $\mathrm{p}$ (AMPS/ CholNp) and $\mathrm{p}(\mathrm{AMPS} / \mathrm{Np}$ ) were found to be similar to each other; $I_{\mathrm{e}} / I_{\mathrm{m}}=0.16$ and 0.14 for the former and latter, respectively. These observations are an indication that in $\mathrm{p}$ (AMPS/CholNp) the excimer formation is induced by hydrophobic association of the side-chain steroid moieties. A tendency for hydrophobic association of the steroid moieties is sufficiently strong to overcome electrostatic repulsion between AMPS units. In the case of $\mathrm{p}(\mathrm{AMPS} / \mathrm{Np})$, however, electrostatic repulsion between AMPS units prevails over hydrophobic association of naphthalene labels, and hence the formation of excimer is virtually prohibited.

Figure 5 compares $I_{\mathrm{e}} / I_{\mathrm{m}}$ ratios for the naphthalenelabeled polymers, $\mathrm{p}$ (AMPS/CholNp) and $\mathrm{p}$ (AMPS/ $\mathrm{Np}$ ), at $C_{\mathrm{p}}=5.0 \mathrm{~g} / \mathrm{L}$ in water in the presence of varying [SC]. In the case of $\mathrm{p}$ (AMPS/CholNp), the $I_{\mathrm{e}} / I_{\mathrm{m}}$ ratio decreases with increasing $[\mathrm{SC}]$ over the whole range of $[\mathrm{SC}]$ investigated. This observation indicates that SC molecules are bound to the aggregates of hydrophobic groups in $\mathrm{p}$ (AMPS/CholNp), which leads to a disruption of steroid aggregates, leading in turn to a disruption of excimers of naphthalene labels. It is to be noted that the $I_{\mathrm{e}} / I_{\mathrm{m}}$ ratio decreases significantly even when $[\mathrm{SC}]<\mathrm{cmc}$ (i.e., ca. $10 \mathrm{mM}$ ) but the

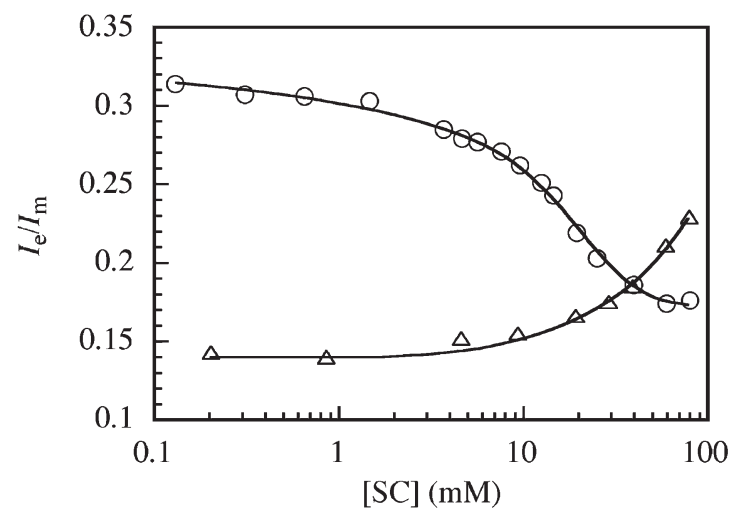

Figure 5. Intensity ratios of excimer and monomer emissions $\left(I_{\mathrm{e}} / I_{\mathrm{m}}\right)$ for naphthalene labels in $\mathrm{p}(\mathrm{AMPS} / \mathrm{CholNp})(\bigcirc)$ and $\mathrm{p}(\mathrm{AMPS} / \mathrm{Np})(\triangle)$ plotted as a function of $[\mathrm{SC}]$.

decrease becomes more significant as $[\mathrm{SC}]$ is increased beyond $\mathrm{cmc}$, reaching a minimum value of 0.17 at $[\mathrm{SC}]=60 \mathrm{mM}$. Each naphthalene label may be incorporated into the micelles formed from SC separately. In the case of $\mathrm{p}$ (AMPS/Np), the $I_{\mathrm{e}} / I_{\mathrm{m}}$ ratios are virtually independent of $[\mathrm{SC}]$ when $[\mathrm{SC}]$ is very low $(<1 \mathrm{mM})$, but it increases slightly with increasing $[\mathrm{SC}]$ in the region where $[\mathrm{SC}]>10 \mathrm{mM}$. This tendency of $\mathrm{p}(\mathrm{AMPS} / \mathrm{Np}$ ) is completely opposite to that of $\mathrm{p}$ (AMPS/CholNp). There are two plausible explanations for the slight increase in the $I_{\mathrm{e}} / I_{\mathrm{m}}$ ratio for the reference polymer as follows: (1) As micelles are formed from $\mathrm{SC}$ at $[\mathrm{SC}]>\mathrm{cmc}$, naphthalene labels in the reference polymer may be preferably incorporated into the micelle. When micelle concentrations are sufficiently high, two or more naphthalene labels may be incorporated in the same micelle, thus leading to the formation of excimer in the micelle. (2) As the concentration of SC is increased, the polymer chain adopts a more compact conformation because of an increase in the ionic strength, and hence the excimer formation becomes more favorable. We added $\mathrm{NaCl}$ in place of SC and confirmed that there was only a minor effect of ionic strength. Therefore, we concluded that the former contribution is much larger than the latter for the slight increase in the $I_{\mathrm{e}} / I_{\mathrm{m}}$ ratio for the reference polymer.

A hypothetical model for the interaction between $\mathrm{p}$ (AMPS/Chol) and SC is illustrated in Figure 6. An important finding in this work is that $\mathrm{p}(\mathrm{AMPS} / \mathrm{Chol})$ can take up SC in water at very low concentrations below its cmc. Therefore, it may be expected that the cholesterol-bearing amphiphilic polyelectrolyte acts as an efficient adsorbent for bile salts. The cholesterol-bearing polymer affects the physiological behavior of bile salts. The cholesterol-bearing polyelectrolyte may reduce the reabsorption of bile salts in ileum, leading to an increased excretion of bile salts. Therefore, this restrains the hepatic conversion of bile 

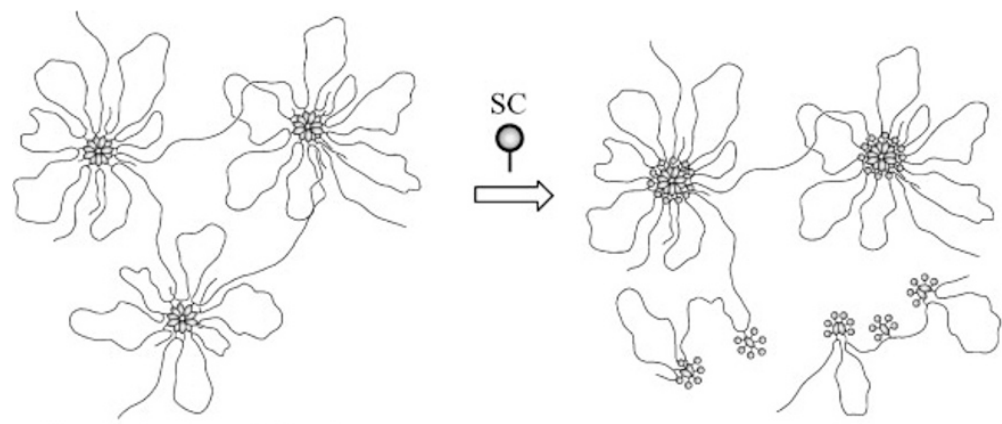

Intermoleculary bridged flower micelle

Figure 6. Conceptual illustration of the binding of SC to intermolecularly bridged flower micelles formed from p(AMPS/Chol) leading to a disruption of the micelle.

acids to cholesterol, and consequently may reduce cholesterol level in blood.

\section{CONCLUSIONS}

The proton NMR spin-spin relaxation time data indicated that SC interacted with the pendent cholesteryl moieties of $\mathrm{p}$ (AMPS/Chol) below the $\mathrm{cmc}$ of SC. SLS and QELS data showed that the intermolecularly bridged flower micelles formed from p(AMPS/ Chol) were partially disrupted by the addition of SC to the aqueous solution. The fluorescence spectra for the naphthalene label of $\mathrm{p}$ (AMPS/CholNp) suggested that the hydrophobic microdomains formed from the pendent cholesteryl moieties of the copolymers were disrupted by the addition of SC.

\section{REFERENCES}

1. A. F. Hofmann and D. M. Small, Annu. Rev. Med., 18, 333 (1967).

2. M. C. Carey and W. C. Duane, in "The Liver: Biological and Pathobiology," I. M. Arias, J. L. Boyer, and N. Fausto, Eds., Raven Press, New York, N.Y., 1994.

3. M. Dumont, S. Erlinger, and S. Uchman, Gastroenterology, 78, 82 (1980).

4. F. A. Wilson, Am. J. Physiol., 241, G83 (1981).

5. P. L. Dubin, D. R. Rigsbee, L. M. Gan, and M. A. Fallon, Macromolecules, 21, 2555 (1988).

6. F. M. Winnik, H. Ringsdorf, and J. Venzmer, Langmuir, 7, 905 (1991).

7. M. Mizusaki, Y. Morishima, K. Yoshida, and P. L. Dubin, Langmuir, 13, 6941 (1997).

8. M. A. Strege, P. L. Dubin, J. S. West, and F. C. D. Daniel, in "Protein Purification; from Molecular Mechanisms to Large-Scale Process," M. Ladisch, R. C. Willson, C. C. Painton, and S. E. Builder, Eds., American Chemical Society, Washington, D.C., 1990.

9. A. D. Read, Polym. J., 4, 253 (1972).

10. S. Yusa, M. Kamachi, and Y. Morishima, Langmuir, 14, 6059 (1998).

11. S. Yusa, A. Hashidzume, and Y. Morishima, Langmuir, 15, 8826 (1999).

12. S. Yusa, K. Ikeda, T. Yamamoto, and Y. Morishima, Polym. J., 34, 851 (2002).

13. H. G. Elias, J. Macromol. Sci., Part A, 7, 601 (1973).

14. Y. Chang and J. R. Cardinal, J. Pharm. Sci., 67, 174 (1978).

15. J. Zakrzewska, V. Markovic, D. Vucelic, L. Feigin, A. Dembro, and L. Mogilevsky, J. Phys. Chem., 94, 5078 (1990).

16. H. Gustavsson and B. Lindman, J. Am. Chem. Soc., 97, 3923 (1975).

17. K. W. Yeo, C. H. Chew, L. M. Gan, L. L. Koh, and H. H. Teo, J. Macromol. Sci., Chem., A26, 663 (1989).

18. S. Meiboom and D. Gill, Rev. Sci. Instrum., 29, 688 (1958).

19. J. Jakes, Czech. J. Phys., B38, 1305 (1988).

20. K. Schillén, W. Brown, and R. M. Johnsen, Macromolecules, 27, 4825 (1994).

21. W. Brown, K. Schillén, M. Almgren, S. Hvidt, and P. Bahadur, J. Phys. Chem., 95, 1850 (1991).

22. B. J. Berne and R. Pecora, in "Dynamic Light Scattering," John Wiley \& Sons, New York, N.Y., 1976.

23. C. S. Johnson and D. A. Gabriel, in "Laser Light Scattering," Dover Publications, New York, N.Y., 1995.

24. D. M. Small, Adv. Chem. Ser., 84, 31 (1968).

25. Y. C. Chiu and K. M. Yu, J. Dispersion Sci. Technol., 13, 587 (1992).

26. M. Ueno, Y. Kimoto, Y. Ikeda, H. Momose, and R. Zana, J. Colloid Interface Sci., 117, 179 (1987).

27. A. Norman, Acta Chem. Scand., 14, 1295 (1960). 